\title{
Efficient Trajectory Options Allocation for the Collaborative Trajectory Options Program
}

\author{
Olga Rodionova, Heather Arneson \\ and Banavar Sridhar \\ NASA Ames Research Center, \\ Moffett Field, Mountain View, CA, USA
}

\author{
Antony Evans \\ CCI-USRA/NAMS, \\ NASA Ames Research Center, \\ Moffett Field, Mountain View, CA, USA
}

\begin{abstract}
The Collaborative Trajectory Options Program (CTOP) is a Traffic Management Initiative (TMI) intended to control the air traffic flow rates at multiple specified Flow Constrained Areas (FCAs), where demand exceeds capacity. CTOP allows flight operators to submit the desired Trajectory Options Set (TOS) for each affected flight with associated Relative Trajectory Cost (RTC) for each option. CTOP then creates a feasible schedule that complies with capacity constraints by assigning affected flights with routes and departure delays in such a way as to minimize the total cost while maintaining equity across flight operators. The current version of CTOP implements a Ration-by-Schedule (RBS) scheme, which assigns the best available options to flights based on a First-Scheduled-FirstServed heuristic. In the present study, an alternative flight scheduling approach is developed based on linear optimization. Results suggest that such an approach can significantly reduce flight delays, in the deterministic case, while maintaining equity as defined using a Max-Min fairness scheme.
\end{abstract}

Keywords-Collaborative Trajectory Options Program (CTOP), Collaborative Decision Making (CDM), Traffic Management Initiative (TMI), Flow Constrained Area (FCA), Trajectory Options Set (TOS), Ration-by-Schedule (RBS), Mixed-Integer Linear Program (MILP), efficiency and equity trade-off, Max-Min fairness

\section{INTRODUCTION}

Traffic Flow Management (TFM) in the National Airspace System (NAS) is in charge of balancing demand and capacity [1]. Severe, and in particular convective weather, significantly reduces the airspace capacity and causes air traffic disruptions and delays in the NAS $[2,3]$. In order to regulate the traffic flow when demand is expected to exceed capacity, Traffic Management Initiatives (TMIs) are implemented [1,4]. Some of the most commonly used TMIs are Ground Delay Programs (GDPs) and Airspace Flow Programs (AFPs).

A GDP is aimed at controlling the arrival rate at an affected airport by assigning departure delays to flights at their origin airports. An AFP is designed to control a flow rate through a certain Flow Constrained Area (FCA) defined in accordance with the convective weather forecast affecting the airspace capacity and the expected demand. Similar to GDPs, AFPs assign departure delays to flights scheduled to pass through the FCA in order to meet the capacity requirement. In addition to this, AFPs specify available reroutes that avoid the FCA. Flight operators may then choose to accept the delay for an affected flight, or to take the available reroute.
In order to increase user involvement and flexibility in route selection, the Collaborative Decision Making (CDM) initiative recently developed a new TMI, the Collaborative Trajectory Options Program (CTOP) [5]. CTOP combines the capabilities of GDPs and AFPs by controlling the flow rates at multiple defined FCAs and/or multiple constrained airports within the same program [6]. Similar to AFPs, CTOP assigns departure delays and reroutes to flights captured by the program. However, unlike AFPs, CTOP allows flight operators to submit a set of desired route options for affected flights, a Trajectory Options Set (TOS), with pre-calculated Relative Trajectory Cost (RTC) associated with each trajectory option. Each impacted flight is then assigned a route from its TOS, and an Expected Departure Clearance Time (EDCT).

In the existing implementation of CTOP, route and delay assignment is performed via a Ration-by-Schedule (RBS) scheme, which is based on the First-Scheduled-First-Served principle [6,7]. RBS is considered to be equitable by flight operators, as the flights are treated in the order they are initially scheduled. However, RBS allocation in the case of multiple consecutive FCAs is usually far from optimal, with respect to overall system cost. The aim of the present study is to develop a more efficient approach to flight scheduling within CTOP based on optimization.

The paper is organized as follows. In Section II, CTOP is introduced, and the TOS allocation problem is stated. Section III summarizes literature focused on resource allocation problems in TFM. In Section IV, two applications of the RBS scheme are described, and a Mixed-Integer Linear Program (MILP) mathematically modeling the TOS allocation problem is formulated. Section V presents simulation results for a sample problem comparing the MILP and the RBS approaches. Finally, this work is summarized and ideas for future work are discussed in Section VI.

\section{CTOP DESCRIPTION AND PROBLEM STATEMENT}

CTOP aims at generating safe and efficient route allocation and flight scheduling under the airspace constraints, taking into account the preferences of flight operators. For such problems within CDM, typically four main criteria are considered [8]:

- System efficiency, which is concerned with reducing flight costs, minimizing delays, and maximizing throughput; 
- Equity, which requires the resource allocation to be fair towards all users (players);

- Flexibility, which is concerned with allowing flight operators to transfer the resources allocated to them by the TFM between their flights;

- Predictability, which requires the solution to be robust towards possible system uncertainties.

CTOP is a complex program which targets all of these criteria and requires several steps to be accomplished by the TFM and the flight operators in order to obtain an efficient and safe flight schedule [6,7]. These include:

- Identifying FCA positions, program start time and duration, and FCA capacities (e.g. [9]);

- Identifying the flights captured by the program, as well as exempt flights;

- Identifying TOSs for the captured flights and their costs;

- Allocating routes to flights and setting the EDCTs;

- Automatic revision (compression) due to possible cancellations $[10,11]$ and flight substitutions performed by flight operators [12]; scheduling pop-up flights [10].

FCAs are typically defined as lines in the airspace through which traffic flow rates should be controlled. They often follow sector boundaries and can consist of several segments. In this paper, for simplicity, the term "FCA" refers to any kind of constrained resource, including en-route FCAs, airport runways and meter-fixes. In contrast to GDPs and AFPs, each of which implements a single FCA as a constraint, CTOP can incorporate multiple, and in particular, consecutive FCAs. Two examples demonstrating the usefulness of this feature are shown in Fig. 1. In Fig. 1, left, a flight from Cleveland Hopkins International Airport (CLE) to Newark Liberty International Airport (EWR) first crosses an area with forecast convective weather where FCAA01 is put in place, and then lands at EWR, which also has reduced capacity due to weather. In Fig. 1, right, EWR has reduced capacity and one of its three meter-fixes (the north one, SHAFF) is affected by convective weather, while the other two are clear. Two independent FCAs can be considered in this case: at SHAFF, and at EWR. Any flight arriving to EWR through SHAFF would cross these two FCAs.

Once the FCAs are defined, the TFM identifies the flights that are planned to fly through (or land at) these FCAs. However, some flights, including flights that are already in the air when the program is issued, international flights, long-haul flights which depart far from the considered FCAs, and flights included in any higher priority TMI are typically exempted from the program. They are assigned the airspace resources on a FirstCome-First-Served (FCFS) basis and further reduce airspace capacity available for non-exempted flights, which presents an important issue from the equity perspective [13].

All non-exempted flights are included in the CTOP, and flight operators are then advised to submit a TOS of their choice for each of these flights. Such route options can be selected from commonly used routes (e.g. Playbook Routes [14], Coded Departure Routes [15]), extracted from historic data (e.g. [16]),
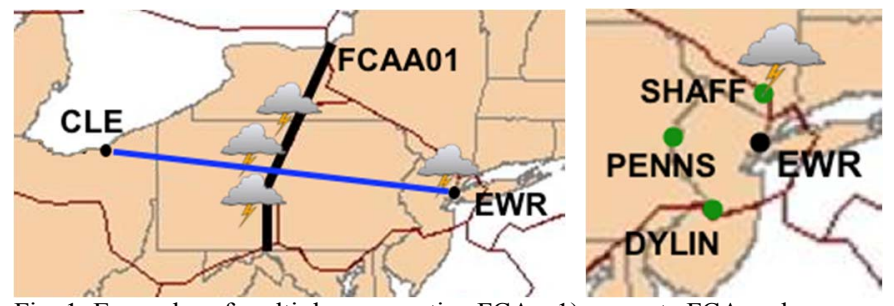

Fig. 1. Examples of multiple consecutive FCAs: 1) en-route FCA and an airport with reduced capacity (left); 2) arrival meter-fixes and an airport with reduced capacities (right)

or created using any approach validated by the flight operator (e.g. [17-20]). Flight operators then assign RTCs to these routes which are calculated using the flight operator preferred cost function (e.g. [21-23]) and translated into their equivalent in departure delay minutes [7]: the RTC of any secondary option is equal to the maximum departure delay that the flight is willing to accept in order to use its preferred route option rather than taking this secondary one.

TFM receives the TOSs submitted by the flight operators for each of their affected flights, and performs allocation of the route options and departure delays to these flights so as to satisfy the option preferences and FCA capacity constraints [6,7]. The present paper focuses particularly on this step, which is reduced to a resource allocation problem. The following input data is considered given:

- A set of FCAs with their positions and capacities over time, with program start and end times;

- A set of affected flight operators and a set of their nonexempt flights captured by the program;

- A TOS for each of the considered flights with RTCs, where these routes may go through any combination of defined FCAs (including avoiding them all).

The present research focuses on two allocation criteria: system efficiency and equity. It is assumed that flexibility is provided via flight swapping at a latter step of CTOP [10-12]. Predictability, which is also an important issue, will be considered in future work.

\section{RESOURCE AlLOCATION STRATEGIES: BACKGROUND}

When addressing a resource allocation problem, one has to define (1) what resources must be allocated, (2) what allocation principles and criteria are to be used, and (3) which allocation algorithm is to be used. This section first presents an overview of existing methods based on how they translate FCA capacities into resource constraints, and then discusses different approaches to address efficiency and equity criteria in TMIs.

\section{A. Different types of resources in TMIs}

The main resources which the flights and the flight operators compete for in CTOP are the FCA capacities. In the literature, there are three common approaches to transform these physical capacities into mathematical constraints: capacity-based, slotbased, and space-based resource allocation.

In capacity-based allocation, constraints are imposed on the number of aircraft permitted in the restricted airspace within a certain period of time (typically, 15 minutes) [8,17-20,25,26]. 
This approach supports multiple constrained resources within a large-scale environment and can be easily extended to a stochastic formulation with varying capacities and demand [27]. However, capacity-based allocation does not support distribution of flights within the time periods with defined capacities [26]. As a result, under such an allocation, flights are most likely to be concentrated at the beginning of each time period, as shown in Fig. 2. A related approach is aggregate modelling of air traffic flows [28-31], where the airspace is modelled as a network with node and link capacities, and traffic demand is aggregated into flows through this network. However, as the variables defined in aggregate flow models are flow variables, a disaggregation approach is required to assign routes and delays to individual flights [28,31].

In slot-based allocation, each time interval at each FCA is subdivided into equal time slots, with each slot assigned to at most one flight. The number of slots and slot duration depend on the FCA capacities. The resource allocation problem is therefore reduced to a slot assignment problem and is typically formulated with binary decision variables associating slots with flights [7,10-13,32-35]. Slot-based allocation is the approach used in GDPs, AFPs and CTOP [6,7,10]. It yields uniform flight distribution within the given time period, as shown in Fig. 3. Thus, slot-based allocation produces more operationally acceptable results than capacity-based allocation. However, stochastic formulation of the slot assignment problem including varying capacities is computationally intensive, as the number of decision variables is directly related to the number of slots.

In space-based allocation, the flights are scheduled so as to maintain a minimum spacing at the boundary of the constrained resources, expressed in terms of time or distance, and defined in such a way as to satisfy the resource capacity. Space-based scheduling is currently implemented in TMIs such as Miles-inTrail (MIT), Minutes-in-Trail (MINIT), and Time-Based Flow Management (TBFM), typically used to apportion traffic into manageable flows and to provide spacing for additional traffic [5]. To the authors' knowledge, space-based allocation has not been applied to problems associated with GDPs, AFPs or CTOPs. This may be because it is deemed too restrictive for large FCAs (such as shown in Fig. 1, left, and Fig. 4), as there is no need to enforce spacing between flights that cross the FCA at

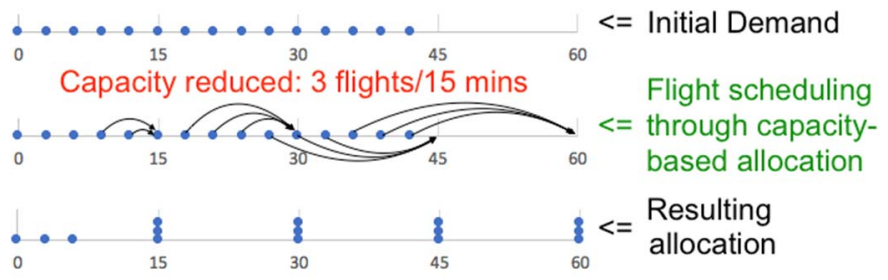

Fig. 2. Example of flight scheduling through capacity-based allocations: initial traffic demand is 5 flights/ 15 minutes and uniform (top); after the capacity is reduced to 3 flights $/ 15$ minutes a reallocation is made (middle), resulting in 3

flights being scheduled at the beginning of each 15-munutes interval (bottom).

$$
\begin{aligned}
& \begin{array}{l}
\text { Capacity: } 3 \text { flights } / 15 \text { minutes } \\
=3 \text { slots } / 15 \text { minutes }
\end{array} \\
& =>\text { slot size }=5 \text { minutes } \\
& \text { slot-based allocation }
\end{aligned}
$$

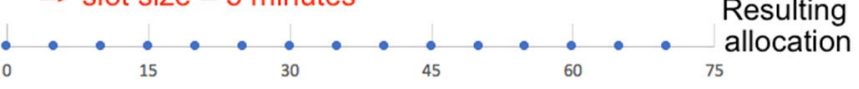
are evenly spaced within each 15-minutes time-interval. different locations. However, large FCAs can be subdivided into smaller FCAs based on dominant traffic flows. It is likely that this is how CTOP will be implemented. Thus, space-based allocation is targeted at flights that cross the FCA at roughly the same location.

Given deterministic constraints, solutions found using spacebased and slot-based allocations are very similar (e.g., for the problem from Fig. 2, space-based allocation produces the same results as shown in Fig. 3), since the time-based spacing is equal to the slot size for the same problem (both are deduced from the resource capacity). However, space-based allocation is better suited than slot-based allocation for stochastic optimization problems that arise when considering uncertain constraints, as these uncertainties are incorporated into the problem parameters and do not affect the number of decision variables. While this work is focused on deterministic formulation, future work will account for the uncertainty in both capacity and demand. Thus, the solution methods of this work were developed with the intent of extending them to the stochastic problem, and the authors chose to implement the space-based allocation in both RBS and MILP formulations introduced in Section IV.

\section{B. Efficiency and equity trade-off in TMIs}

Appropriate metrics are required to compare the performance of different algorithms. As stated above, this paper seeks to quantify system efficiency and equity. System efficiency is related to the cost incurred by the system users as a result of the allocation. Some measures of system efficiency typically considered in literature are total ground delay [13,19,25,35], airborne delay $[17,18,20]$ and arrival delay [21,22] for flights; total arrival delay for passengers [22,23]; the number of flights arriving on-time [36]; flight cancellation cost [21]; cost of taking non-preferred route [19,20,21,34]; throughput at the FCAs [23]. For simplicity, system efficiency is addressed in this paper by minimizing the total flight ground and airborne delays, plus the RTCs of the selected route options from TOSs (with corresponding scaling factors, as described in Section IV-C).

While it can be clearly demonstrated that submitting alternative route options improves system efficiency [23-25], there has been hesitancy to do so in practice, possibly because of equity concerns. Indeed, some studies $[10,35,37,38]$ show that gaming may be an issue in CTOP. Thus, it is critical to consider equity within allocation. In literature, multiple equity criteria have been proposed [39], however, no one of them is universally accepted. Numerous studies address equity by defining the flight priority order, which is considered to be equitable from the TFM and flight operators perspective, and scheduling flights in this order using FCFS $[8,13,17,24,33]$. One of the most commonly used schemes is RBS [7,10,11], currently implemented in GDPs, AFPs and CTOP. It orders flights based on their Estimated Times of Arrival (ETAs) at the FCAs as scheduled initially, which is considered to be equitable by airlines. RBS is proven to always yield the solution minimizing the total system delays in the case of a single-resource problem (like a GDP or an AFP) [11]. However, this is no longer true in the case of multi-resource problems, like CTOP [40]. Thus, some other studies choose to address equity by solving an optimization problem which minimizes system costs and, at the same time, the deviation of the resulting flight order from the RBS order [26,36,40]. 
Alternatively, some other works do not consider a particular flight order to be equitable by default, and appeal instead to equity theory arising from economics studies [22,29,32,38,39]. A common measure of equity in economics is the minimum individual derived utility among all players [22]. According to this measure, the most equitable allocation is provided by the Max-Min fairness scheme [38,39], based on maximizing the minimum derived utility over all players. It is also typically simple to evaluate [38]. Thus, in the present study, equity is quantified using Max-Min fairness, with the maximum average airline cost being chosen as a metric. Average cost was preferred over total cost in order to favor an equitable balance between large and small airlines. However, as demonstrated in [39], allocation under pure Max-Min fairness results in a significant decrease in system efficiency. A trade-off between efficiency and equity metrics is established in this work by introducing weighting coefficients, as described in Section IV-C.

\section{Route AllocAtion APPROACHES: DEVELOPMENT}

This section describes the developed approaches supporting space-based allocation of routes and delays to flights within the CTOP problem: an RBS scheme and its adaptation to multipleFCAs environment, and a MILP simultaneously optimizing system efficiency and equity.

\section{A. Input data for the CTOP problem}

Input data for the resource allocation problem in the context of CTOP is defined below. It is assumed that each FCA (including en-route FCAs, airports, sector boundaries, etc.) can be defined within a sequence of time periods, with different capacities associated with each period. The following data is then considered for the FCAs:

- $\quad Z$ - total number of FCAs within a CTOP;

- $\quad L^{k}$ - number of time periods defined for FCA $k$;

- $S^{k, l}-$ start time of time period $l$ of FCA $k$;

- $\quad E^{k, l}$ - end time of time period $l$ of FCA $k$;

- $\quad V^{k, l}$ - capacity of FCA $k$ within time period $l$;

- $s^{k, l}$ - minimum-time spacing imposed between flights at FCA $k$ within time period $l$ (deduced from $V^{k, l}$ );

where $k=1, \ldots, Z$ and $l=1, \ldots, L^{k}$. If the capacity is given as a number of flights per hour, then $s^{k, l}=60 / V^{k, l} \mathrm{~min}$. In order to assure the connectivity of the time periods, the start and end time should match: $S^{k, l}=E^{k, l-1}$, for $l=2, \ldots, L^{k}$. To simplify the formulation, two additional time periods are added before and after FCA actual period of activity, defined by the parameters:

- $S^{k, 0}=0, E^{k, 0}=S^{k, 1}$ - time period before FCA $k$ becomes active;

- $S^{k, L^{k}+1}=E^{k, L^{k}}, E^{k, L^{k}+1}=M$ - time period after FCA $k$ is deactivated;

- $s^{k, 0}=s^{k, L^{k}+1}=-2 M$,

where $M$ is some large constant. Negative spacing is equivalent to no spacing: in the present study, flights arriving before or after the FCA period of activity are not required to be spaced.
Acceptable spacing can be achieved by setting a maximum available capacity for these bounding time periods.

The flight data includes only non-exempted flights captured by the program, and is defined as follows:

- $\quad N$ - number of flights;

- $\quad N^{A}$ - number of airlines;

- $\Lambda^{u}=\left\{i_{u}^{1}, i_{u}^{2}, \ldots, i_{u}^{N^{u}}\right\}-$ a set of indices of flights of airline $u, N^{u}=\left|\Lambda^{u}\right|$;

- $\quad N_{i}$ - number of TOS options for flight $i$;

- $\quad q_{i j}-\mathrm{RTC}$ of $j$-th route (option) of flight $i$;

- $\Omega_{i j}=\left(k_{i j}^{1}, k_{i j}^{2}, \ldots, k_{i j}^{H_{i j}}\right)-$ an ordered set of indices of FCAs along route $j$ of flight $i, H_{i j}=\left|\Omega_{i j}\right|$;

- $t_{i j}^{k}$ - ETA of flight $i$ at FCA $k$ along route $j, k \in \Omega_{i j}$;

- $\quad \widetilde{\Omega}_{i}=\left\{k_{i}^{1}, k_{i}^{2}, \ldots, k_{i}^{\widetilde{H}_{i}}\right\}-$ a set of indices of FCAs which flight $i$ may cross along any of its routes $j=1, \ldots, N_{i}$; $\widetilde{\Omega}_{i}=\bigcup_{j=1}^{N_{i}} \Omega_{i j}, \widetilde{H}_{i}=\left|\widetilde{\Omega}_{i}\right|$;

- $\Phi_{i}^{k}=\left\{j_{k i}^{1}, j_{k i}^{2}, \ldots, j_{k i}^{\mathrm{F}_{i}^{k}}\right\}-\mathrm{a}$ set of indices of routes of flight $i$ which are planned to cross FCA $k, \mathrm{~F}_{i}^{k}=\left|\Phi_{i}^{k}\right|$;

- $\widetilde{\Phi}^{k}=\left\{i_{k}^{1}, i_{k}^{2}, \ldots, i_{k}^{k}\right\}-$ a set of indices of flights which may cross FCA $k$ along any of its route, $\tilde{F}^{k}=\left|\widetilde{\Phi}^{k}\right|$;

where $u=1, \ldots, N^{A} ; i=1, \ldots, N ; j=1, \ldots, N_{i}$ and $k=1, \ldots, Z$. In the ordered set $\Omega_{i j}$, index $k_{i j}^{h}$ precedes index $k_{i j}^{h+1}$, if route $j$ of flight $i$ crosses FCA $k_{i j}^{h}$ prior to FCA $k_{i j}^{h+1}$. Some of the sets $\Omega_{i j}$ and $\Phi_{i}^{k}$ may be empty. Fig. 4 shows an example of the input data definition for a flight $(i=1)$ from Nashville International Airport (BNA) to EWR. Here, three FCAs are involved in a CTOP: FCAs 1 and 2 correspond to canonic FCAA04 and FCAA01 respectively, and FCA 3 ensures capacity restrictions at EWR. Four options are defined for this flight: route 1 passes through all three FCAs $\left(\Omega_{11}=\{1,2,3\}\right)$; routes 2 and 3 pass

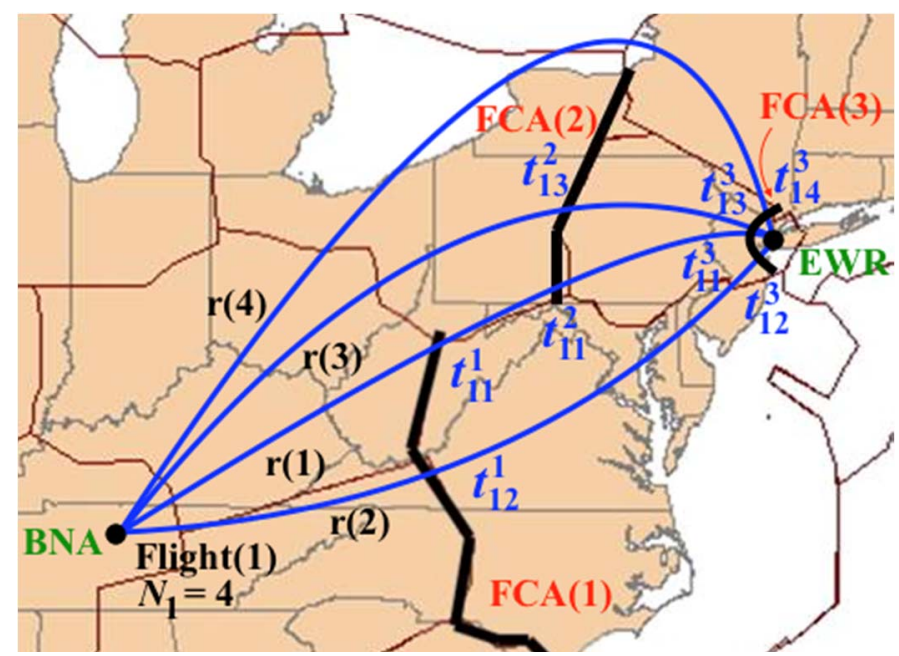

Fig. 4. TOS example: four trajectory options for a flight from BNA to EWR with ETAs at the three FCAs. 
through only one of the two en-route FCAs, and the FCA at $\operatorname{EWR}\left(\Omega_{12}=\{1,3\}\right.$, and $\left.\Omega_{13}=\{2,3\}\right)$; finally, route 4 avoids both en-route FCAs $\left(\Omega_{14}=\{3\}\right)$. For each of the routes the ETAs at each of the crossed FCAs are assumed to be given.

The resource allocation problem under CTOP is reduced to allocation of routes from TOSs and delays. Since ground delay is less expensive than airborne delay, the current CTOP implementation assigns only pre-departure (ground) delays, which are then translated into EDCTs. However, in problems involving consecutive FCAs, it may be advantageous to delay flights in the air between consecutive FCAs to control arrival rates at downstream FCAs. Airborne delays may be applied at each FCA $k$ involved in the route $j$ of flight $i\left(k \in \Omega_{i j}\right)$, but they are not necessary at the first FCA, $k_{i j}^{1}$, as such delays can be absorbed on the ground at a lower cost. The following decision variables are therefore introduced for this problem for each flight $i=1, \ldots, N$ and for each its route $j=1, \ldots, N_{i}$ :

- $\quad \delta_{i j}=\left\{\begin{array}{l}1, \text { if route } j \text { is chosen by flight } i \\ 0, \text { otherwise }\end{array} ;\right.$

- $\quad d_{i j} \geq 0-$ ground delay of flight $i$ at route $j$;

- $\quad a_{i j}^{k} \in\left[0, A_{i j}^{k}\right]$ - airborne delay of flight $i$ at route $j$ at FCA $k$, for $k \in \Omega_{i j}$, and $k \neq k_{i j}^{1}$;

where parameters $A_{i j}^{k}$ define the maximum amounts of delay which a flight can absorb in the air, and depend on the aircraft type, speed, flying distance and airspace availability. The methods to set the values for these decision variables taking into account the problem constraints are discussed below.

\section{B. Ration-by-Schedule scheme}

In the current version of CTOP, flight scheduling by RBS is implemented through slot-based allocation $[6,7,11]$. In this section, the adaptation of this method to the space-based allocation is presented. It requires the following steps.

1. For each flight $i$, its Initial Arrival Time (IAT) is calculated, i.e., the earliest ETA at its first, or the closest, FCA included in its TOS: $I A T_{i}=\min _{j=1, \ldots, N_{i}}\left\{t_{i j}^{k_{i j}^{1}}\right\}$, where $k_{i j}^{1} \in \Omega_{i j}$ is the first FCA along the route $j$.

2. Flights are ordered in the priority list based on their IATs: $\left(i_{1}, i_{2}, \ldots, i_{N}\right)$, so that $I A T_{i_{p}} \leq I A T_{i_{p+1}}$.

3. Flights are pulled one by one from the priority list. For each next flight $i$, the total cost of each its route $j=1, \ldots, N_{i}$ is calculated as follows.

3.1. The primary FCA, i.e. the first FCA along route $j$, is identified, denoted henceforth as $k$, i.e. $k \equiv k_{i j}^{1}$, where $k_{i j}^{1} \in \Omega_{i j}$ (if $\Omega_{i j}=\emptyset$, step 3.2 is omitted).

3.2. The minimum ground delay, $d_{i j}$, is calculate in such a way as to maintain the required spacing $s^{k, l}$ at FCA $k$ between flight $i$ and all flights already scheduled through this FCA: $\left|t_{i j}^{k}+d_{i j}-T_{f}^{k}\right| \geq s^{k, l}$, where $T_{f}^{k}$ is the ETA of another flight $f$ scheduled through FCA $k$ at a previous step.
3.3. The total cost of route $j$ is calculated: $c_{i j}=d_{i j}+q_{i j}$.

4. The least-cost route, $j^{*}$, such that $c_{i j^{*}}=\min _{j=1, \ldots, N_{i}}\left\{c_{i j}\right\}$, is assigned to flight $i$, and the following steps are made.

4.1. Ground delay $d_{i}^{*} \equiv d_{i j^{*}}$ is assigned to flight $i$.

4.2. EDCT for flight $i$ is calculated by adding the ground delay $d_{i}^{*}$ to the initial departure time.

4.3. The ETA of flight $i$ at the primary FCA along route $j^{*}, k_{i}^{*} \equiv k_{i j^{*}}^{1} \in \Omega_{i j^{*}}$, is calculated and added to the list of ETAs for this FCA: $T_{i}^{k_{i}^{*}}=t_{i j^{*}}^{k_{i}^{*}}+d_{i}^{*}$.

5. The ETAs of flight $i$ at all secondary FCAs (subsequent to FCA $k_{i}^{*}$, if any) are adjusted. The total flight delay, $d_{i}$, is set to equal the ground delay: $d_{i}=d_{i}^{*}$, and for each subsequent FCA $k_{i j^{*}}^{h} \in \Omega_{i j^{*}}, h=2, \ldots, H_{i j^{*}}$, denoted henceforth as $k$, i.e. $k \equiv k_{i j^{*}}^{h}$, the following steps are performed.

5.1. The new total flight delay, $\tilde{d}_{i}$, is calculated in such a way as to maintain required spacing $s^{k, l}$ between flight $i$ and all flights already scheduled through FCA $k: \tilde{d}_{i} \geq d_{i}$ and $\left|t_{i j^{*}}^{k}+\tilde{d}_{i}-T_{f}^{k}\right| \geq s^{k, l}$ (similar to step 3.2).

5.2. The ETA of flight $i$ at FCA $k$, is calculated and added to the list of ETAs for this FCA: $T_{i}^{k}=t_{i j^{*}}^{k}+\tilde{d}_{i}$ (similar to step 4.3).

5.3. The total flight delay is adjusted: $d_{i}=\tilde{d}_{i}$. Steps 5.15.3 are repeated for all secondary FCAs.

6. Steps 3-5 are repeated until all flights are scheduled.

Through such an allocation, the first flight from the list receives its best option, the next flight receives its best option subject to the choice of the first flight, and so on. As can be seen from step 3 of the algorithm, the classical RBS scheme ignores possible airborne delays induced by the schedule and selects the best options taking into consideration only the constraints at the primary FCA. The reason for this is that, because of the multiple sources of uncertainty in the system at the TFM planning stage, the resulting schedule is highly unlikely to be executed as planned, and exact airborne delays cannot be accurately estimated. In the present paper, an alternative RBS approach is also developed, referred to as RBSall, in which all consecutive FCA constraints are considered at the same time.

RBSall repeats all steps of the classical FCA, except steps 3.1-3.2. Instead of focusing on the primary FCA of route $j$, RBSall considers all FCAs from $\Omega_{i j}$ and calculates the minimum ground delay, $d_{i j}$, in such a way as to satisfy the spacing constraints at all FCAs at the same time: $\left|t_{i j}^{k}+d_{i j}-T_{f}^{k}\right| \geq s^{k, l}$, where $k \in \Omega_{i j}$ and $T_{f}^{k}$ is the ETA of another flight $f$ scheduled through FCA $k$ at a previous step. The new ETAs at the FCAs can then be easily deduced from this delay as: $T_{i j}^{k}=t_{i j}^{k}+d_{i j}$. Step 4.3 of RBSall can be extended to setting the pre-calculated ETAs at all FCAs of the best route $j^{*}$. Step 5 would then no longer be necessary. In some cases, taking into account a sequence of involved FCAs may produce a better schedule than 
considering only the primary FCA, at least in the deterministic conditions, as demonstrated in Section V.

\section{Optimization problem formulation}

As discussed in Section III-B, an alternative approach to sequential scheduling is global optimization. Below, a MILP using the input data and variables defined in Section IV-A is formulated which minimizes total system cost (an efficiency metric) and maximum average airline cost (an equity metric) simultaneously.

$$
\begin{aligned}
& \min _{\delta, d, a, y} \alpha \sum_{i=1}^{N} c_{i}+\omega y \\
& \text { s.t. } c_{i}=\sum_{j=1}^{N_{i}}\left(\beta q_{i j} \delta_{i j}+d_{i j}+\gamma \sum_{h=2}^{H_{i j}} a_{i j}^{k_{i j}^{h}}\right), i=1, \ldots, N \\
& y \geq \frac{1}{N^{u}} \sum_{i \in \Lambda^{u}} c_{i}, \quad u=1, \ldots, N^{A} \\
& \sum_{j=1}^{N_{i}} \delta_{i j}=1 \\
& i=1, \ldots, N \\
& d_{i j}+\sum_{h=2}^{H_{i j}} a_{i j}^{k_{i j}^{h}} \leq M \delta_{i j}, \quad i=1, \ldots, N ; j=1, \ldots, N_{i} \\
& \tau_{i}^{k}=\sum_{j \in \Phi_{i}^{k}}\left(t_{i j}^{k} \delta_{i j}+d_{i j}+\sum_{m \in \Omega_{i j} ; 2 \leq \mathrm{id}(m) \leq \mathrm{id}(k)} a_{i j}^{m}\right), \\
& i=1, \ldots, N ; k \in \widetilde{\Omega}_{i} \\
& \tau_{i}^{k} \geq \sum_{l=0}^{L^{k}+1} S^{k, l} x_{i}^{k, l}, \quad i=1, \ldots, N ; k \in \widetilde{\Omega}_{i} \\
& \tau_{i}^{k}<\sum_{l=0}^{L^{k}+1} E^{k, l} x_{i}^{k, l}, \quad i=1, \ldots, N ; k \in \widetilde{\Omega}_{i} \\
& \sum_{l=0}^{L^{k}+1} x_{i}^{k, l} \leq 1, \quad i=1, \ldots, N ; k \in \widetilde{\Omega}_{i} \\
& M v_{i, f}^{k}+\tau_{i}^{k}-\tau_{f}^{k} \geq \sum_{l=0}^{L^{k}+1} \frac{s^{k, l}}{2}\left(x_{i}^{k, l}+x_{f}^{k, l}\right), \\
& M\left(1-v_{i, f}^{k}\right)+\tau_{f}^{k}-\tau_{i}^{k} \geq \sum_{l=0}^{L^{k}+1} \frac{s^{k, l}}{2}\left(x_{i}^{k, l}+x_{f}^{k, l}\right), \\
& k=1, \ldots, Z ; i, f \in \widetilde{\Phi}^{k} ; i<f
\end{aligned}
$$

$\mathrm{y} \geq 0$

$d_{i j} \geq 0$, $i=1, \ldots, N ; j=1, \ldots, N_{i}$

$0 \leq a_{i j}^{k} \leq A_{i j}^{k}$

$$
\begin{array}{r}
i=1, \ldots, N ; j=1, \ldots, N_{i} ; k \in \Omega_{i j} ; k \neq k_{i j}^{1} \\
\delta_{i j} \in\{0,1\}, \quad i=1, \ldots, N ; j=1, \ldots, N_{i} \\
x_{i}^{k, l} \in\{0,1\}, i=1, \ldots, N ; k \in \widetilde{\Omega}_{i} ; l=0, \ldots, L^{k}+1 \\
v_{i, f}^{k} \in\{0,1\}, \quad k=1, \ldots, Z ; i, f \in \widetilde{\Phi}^{k} ; i<f
\end{array}
$$

The first term of the objective function (1) represents the total cost of the allocation and equals the sum of individual flight costs, defined by (2). The second term introduces a new decision variable, $y$, which stands for the maximum average airline cost. It is defined through constraints (3), where the right-hand-side represents the average cost for airline $u$. Weighting coefficients $\alpha$ and $\omega$ establish the trade-off between system efficiency and equity. Individual flight cost (2) is composed of the RTC $q_{i j}$ for the chosen route (for which $\delta_{i j}=1$ ), the ground delay $d_{i j}$ and the airborne delays $a_{i j}^{k}$ at all the FCAs along the chosen route. Weighting coefficients $\beta$ and $\gamma$ serve to balance these cost components. If RTCs are expressed in minutes of delay, then $\beta=1$. Airborne delays are typically estimated to be twice as expensive as ground delays, thus, most likely, $\gamma=2$.

Constraints (4) ensure that one and only one route is chosen for each flight. Constraints (5) ensure that if a specific route is not chosen, all associated delays along this route are set to zero (here, $M$ is some large constant). With this, as delay is summed up over all possible routes in (2) and (6), only the delay incurred along the flown route is accounted for. Variable $\tau_{i}^{k}$ in (6) represents the new ETA for flight $i$ at FCA $k\left(k \in \widetilde{\Omega}_{i}\right)$. If there exist $j \in \Phi_{i}^{k}$ such that $\delta_{i j}=1$ (the chosen route goes through FCA $k$ ), then $\tau_{i}^{k}$ equals the sum of the initial ETA, $t_{i j}^{k}$, the ground delay, $d_{i j}$, and all airborne delays at the FCAs $m$ preceding the current FCA $k$ on the route $j($ i.e. $\operatorname{id}(m) \leq \operatorname{id}(k))$. If $\delta_{i j}=0$ for all $j \in \Phi_{i}^{k}$ (the chosen route avoids FCA $k$ ), then $\tau_{i}^{k}=0$.

Constraints (7), (8) and (9) serve to identify the time interval where the new ETA of flight $i$ at FCA $k, \tau_{i}^{k}$, falls, and use additional binary variables, $x_{i}^{k, l}$, defined as:

$$
\text { - } x_{i}^{k, l}=\left\{\begin{array}{l}
1, \text { if } S^{k, l} \leq \tau_{i}^{k}<E^{k, l}, \\
0, \text { otherwise }
\end{array},\right.
$$

where $i=1, \ldots, N ; k \in \widetilde{\Omega}_{i}, l=0, \ldots, L^{k}+1$. Constraints (9) ensure that only one of these variables can be non-zero for each flight and each FCA, as $\tau_{i}^{k}$ can belong to only one time interval. Taking (9) into account, constraints (7) and (8) serve to identify the start and end times of this interval, respectively. Finally, (10) and (11) impose spacing constraints at the FCA $k$ for any pair of flights, $i$ and $f$, which are scheduled through this FCA. They arise from the following constraint:

$$
\left|\tau_{i}^{k}-\tau_{f}^{k}\right| \geq \sum_{l=0}^{L^{k}+1} \frac{s^{k, l}}{2}\left(x_{i}^{k, l}+x_{f}^{k, l}\right) .
$$

If both flights $i$ and $f$ are scheduled to arrive at FCA $k$ within its period of activity, i.e., within some time intervals $l_{i}$ and $l_{f}$ respectively, $l_{i}, l_{f} \in\left\{1, \ldots, L^{k}\right\}$, then (18) requires the flight ETAs, $\tau_{i}^{k}$ and $\tau_{f}^{k}$, to be separated by at least a minimum spacing 
averaged between the two intervals: $\frac{1}{2}\left(s^{k, l_{i}}+s^{k, l_{f}}\right)$. If any of these flights is scheduled to arrive at FCA $k$ before or after its period of activity, then (18) is satisfied automatically. Nonlinear constraints (18) are transformed into linear constraints (10) and (11) using additional binary variables, $v_{i, f}^{k}$, defined as:

$$
\text { - } v_{i, f}^{k}=\left\{\begin{array}{l}
1, \text { if } \tau_{f}^{k}-\tau_{i}^{k} \geq 0 \\
0, \text { otherwise }
\end{array}\right.
$$

Constraints (12)-(14) define the boundaries for maximum average airline cost, ground delays and airborne delays; and (15)-(17) define variables $\delta_{i j}, x_{i}^{k, l}$ and $v_{i, f}^{k}$ as binary. Besides the variables from (12)-(17), the MILP (1)-(17) includes additional variables, $c_{i}$ and $\tau_{i}^{k}$, which are introduced to simplify the presentation. These variables can be replaced throughout the formulation with their expressions from (2) and (6) respectively, while (2) and (6) can then be removed from the formulation. For a limited number of FCAs, the total number of variables and constraints in the MILP is of the order of $\sim O\left(N^{2}\right)$.

The presented formulation involves a number of limitations. Generally speaking, a space-based formulation cannot apply sector capacity constraints, in contrast to the capacity-based formulation. It can, however, efficiently handle flows passing across sector boundaries and ensure that these flows are uniformly distributed. Furthermore, for simplicity, the presented MILP does not consider exempted flights. Exemptions can be addressed by reducing the FCAs capacities accordingly, and thus, by increasing the spacing between the non-exempted flights. Alternatively, the exempted flights can be scheduled in a preprocessing stage (applying a FCFS principle, as mentioned in Section II), before being introduced as additional constraints similar to (10) and (11), based on their ETAs. Finally, the presented MILP does not consider pop-up flights. Pop-ups can, however, be handled in the same way as currently done in TMIs: by reserving some capacity for pop-ups and assigning them with average system delay, to support equity.

The presented MILP, further abbreviated as MILP G\&A, includes ground delays and airborne delays before entering each subsequent FCA. Airborne delays can be avoided by setting all associated variable to zero. In this way, a "Ground-Delay-Only" version of the MILP is obtained, abbreviated as MILP GDO.

\section{Simulation Results}

In this section, some simulation results are presented. First, the simulated test case is described. Then, results applying the RBS, RBSall, MILP GDO and MILP G\&A approaches to this test case are compared in terms of efficiency and equity.

\section{A. Test case setup}

To demonstrate the features of the developed routeallocation methods, a simple test case was created for traffic destined for EWR. Traffic data was obtained from the filed flight plan and track information from Aircraft Situation Display to Industry data for July 14, 2015. For each origin-destination pair in this data, a set of possible routes was extracted by clustering flown flight plans (accounting for flight plan amendments) using the techniques described in [16]. The most commonly used route from each cluster was selected and included as a candidate for the TOSs for flights on that origin-destination pair.
Four FCAs were defined: one at the EWR runway threshold and three at the primary EWR arrival meter-fixes (gates): SHAFF (north gate), PENNS (west gate) and DYLIN (south gate), as shown in Fig. 1, right. All FCAs were active for one hour, starting at $0800 \mathrm{Z}$ and ending at 0900Z. Then, for each flight, a TOS was set including the lowest cost routes from those identified in the clustering analysis, intersecting each combination of FCAs, as available. This results in TOSs for each flight with between one and three route options. ETAs were calculated at each FCA crossed by each route option for each flight by averaging actual flight transit times to each FCA for each clustered route over all flights that made up that cluster.

The preferred route (with zero RTC) was then identified as the one with the earliest arrival time at EWR. All flights having any of the preferred route ETAs between $0800 \mathrm{Z}$ and $0900 \mathrm{Z}$ were captured by the CTOP. Among these flights, only those with at least two options per TOS were selected for further simulations in order to diversify the route choices. This resulted in a set of 20 flights from a range of origin airports across the NAS. The TOSs for these flights are shown in Fig. 5, with different colors corresponding to the different arrival gates. The RTC for each option was calculated based on the additional flight time required relative to the preferred route for that TOS, multiplied by a factor of two (a unit of air delay is assumed to be twice as expensive as a unit of ground delay).

Table I summarizes the values considered in this paper for demand, capacity and spacing at the FCAs. Demand was estimated from the selected flights, assuming their preferred routes. To simplify the demonstration of results of the developed techniques, capacities were artificially set equal to demand. As shown further, even this simple case creates an interesting allocation problem enforcing spacing between flights. Recall that these capacities are allocated specifically for the selected flights. The rest of the available capacities are allocated to the exempted flights which are not considered in the present study.

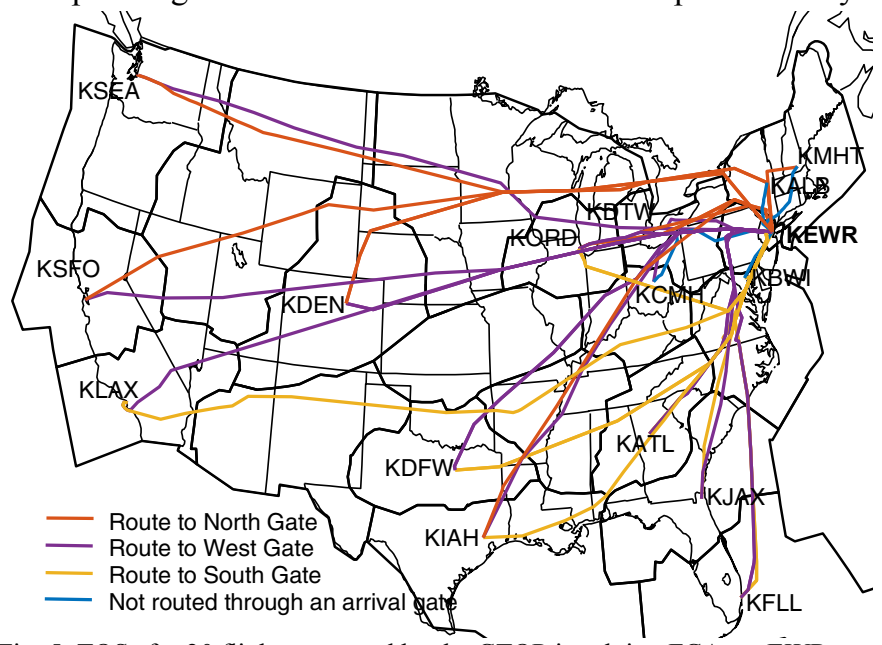

Fig. 5. TOSs for 20 flights captured by the CTOP involving FCAs at EWR and arrival meter-fixes.

TABLE I. INITIAL DEMAND AND CAPACITIES AT FCAS

\begin{tabular}{|l|c|c|c|c|}
\hline & SHAFF & PENNS & DYLIN & EWR \\
\hline Total demand & 4 & 7 & 9 & 20 \\
\hline Demand/active period & 4 & 6 & 6 & 20 \\
\hline Capacity/hour & 4 & 6 & 6 & 20 \\
\hline Spacing, minutes & 15 & 10 & 10 & 3 \\
\hline
\end{tabular}




\section{B. Comparing efficiency of the TOS allocation methods}

In this section, the efficiency of the TOS allocation methods is compared for the described test case while the equity criterion is temporally omitted. The flights are first assigned routes and delays via one of the considered methods, i.e., RBS, RBSall, MILP GDO or MILP G\&A, and then, the Schedule Execution Simulator (SES) is run for the resulting schedules. SES is based on the FCFS scheme and assumes that the flights take off at their assigned EDCTs, follow the assigned routes, and cross the FCAs so as to maintain the required spacing, incurring airborne delays if necessary. SES calculates the total cost of such an execution as the sum of the RTCs, ground delays and airborne delays.

MILP GRO and MILP D\&A were formulated for the four FCAs and 20 flights included in the CTOP. The trade-off coefficients in the objective function (1) were set to $\alpha=1$ and $\omega=0$. Cost-balancing coefficients in (2) were set to $\beta=1$ and $\gamma=2$ (relevant to MILP G\&A only), as stated in Section IV-C. The MILPs were solved using the Gurobi Optimizer (release 7.0.2) for Mac OS X. The RBS schemes and the SES were modeled in Java SE 8u131.

The results of the simulations are summarized in Table II. Here, total calculated cost is the cost yielded by the scheduling method; and total execution cost is the cost yielded by the SES. These costs are equal for all the methods except RBS, as explained in Section IV-B. Total ground cost is the cost associated with the flight on the ground, which includes RTCs and ground delays. Total airborne cost is the difference between the execution cost and the ground cost, which is zero for RBSall and MILP GDO. Throughput is calculated as the number of flights crossing the FCAs within their period of activity (1 hour).

As can be seen from Table II, under RBS, more flights depart on-time than under other methods, which is not surprising, as RBS takes into account only the constraints at the primary FCA. This also results in its total calculated cost being relatively low. However, for this test case, RBS has the highest execution cost, which arises from almost 30 minutes of airborne delays. RBSall slightly outperforms RBS in terms of execution cost, and incurs no airborne delays. It is thus more predictable (in this deterministic case), however, on-time performance and throughput are the worst in this case. MILP GDO yields almost the same calculated cost as RBS, which in this case is not increased during execution. It saves almost 60 minutes of delay compared to RBSall and increases the throughput as well. The

TABLE II. COMPARISON OF SCHEDULING METHODS EFFICIENCY

\begin{tabular}{|l|c|c|c|c|}
\hline & \multicolumn{2}{|c|}{ RBS } & \multicolumn{2}{c|}{ MILP } \\
\cline { 2 - 5 } & Classic & RBSall & GDO & G\&A \\
\hline Total calculated cost, min. & 143 & 195 & 147 & 134 \\
\hline Total execution cost, min. & 201 & 195 & 147 & 134 \\
\hline Total ground cost, min. & 143 & 195 & 147 & 120 \\
\hline Total airborne cost, min. & 58 & 0 & 0 & 14 \\
\hline Maximum flight cost, min. & 22 & 20 & 29 & 35 \\
\hline Max. ground delay, min. & 20 & 20 & 29 & 14 \\
\hline Max. airborne delay, min. & 6 & 0 & 0 & 2 \\
\hline Nb. of on-time departures & 9 & 4 & 4 & 7 \\
\hline Nb. of reroutings & 3 & 2 & 2 & 2 \\
\hline SHAFF throughput & 4 & 2 & 3 & 4 \\
\hline PENNS throughput & 5 & 5 & 6 & 6 \\
\hline DYLIN throughput & 5 & 5 & 5 & 5 \\
\hline KEWR throughput & 14 & 14 & 15 & 16 \\
\hline
\end{tabular}

best results are produced by MILP G\&A. It significantly reduces the ground delay compared to MILP GDO and airborne delay compared to RBS. Moreover, it increases throughput and ontime performance as well. However, in this case, some flights are assigned higher costs than under the RBS. In summary, by taking into account constraints at consecutive FCAs simultaneously, total system cost is reduced while the schedule is also more predictable in execution. Considering airborne delays at the planning stage enables further cost reduction.

It can be noted, however, that all the methods result in significant system delays for a test case in which the initial demand is equal to the available capacity. If scheduling was performed using capacity-based allocation, then the solution would have no delays. However, the space-based allocation imposes the spacing constraints, which are not necessarily satisfied by the initial demand. This is demonstrated in Fig. 6, which displays the arrival sequences of the flights at EWR (with time in minutes). Shaded areas correspond to the time intervals outside the CTOP period of activity. The initial demand at EWR is shown on the left with blue circles. It is non-uniform and does not satisfy the required spacing ( 3 minutes). The 4 scales on the right show the results of the SES applied to the schedules obtained with RBS (yellow), RBSall (green), MILP GDO (red) and MILP G\&A (purple). These flight distributions are more uniform than the initial demand and maintain the required 3minutes spacing. The resulting sequencing is the densest for the MILP G\&A solution, which results in the lowest total delay. Flights that are rerouted from their initial preferred route are highlighted in color. Different methods choose to reroute different flights, and the flight order also differs significantly from one method to another. This results in different flight costs and reveals the equity issues, as shown in the next section.

\begin{tabular}{|c|c|c|c|c|c|}
\hline 555 & & 16 & - $\mathrm{L} 6$ & & \\
\hline 550 & & $\mathrm{Cl}$ & $\begin{array}{l}\mathrm{Cl} \\
\mathrm{U} 2\end{array}$ & : $\begin{array}{l}\mathrm{L} 6 \\
\mathrm{U} 2\end{array}$ & $\bullet \mathrm{Cl}$ \\
\hline 545 & & $\mathbf{U 2}$ & : L7 & 47 & - U2 \\
\hline 540 & L.8 & $\begin{array}{l}51 \\
17\end{array}$ & $\begin{array}{l}51 \\
1.8\end{array}$ & Cl & $\bullet \begin{array}{l}\mathrm{L} 7 \\
\mathrm{~L} 6\end{array}$ \\
\hline $\begin{array}{l}535 \\
530\end{array}$ & ${ }_{1.5}^{\mathrm{Cl} \mathrm{U}^{2}}$ & L.8 & F1 & $\begin{array}{l}\text { L8 } \\
\text { L.5 } \\
\text { - S1 }\end{array}$ & 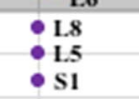 \\
\hline 525 & S1 & F1 & & - $\mathrm{A2}$ & 01 \\
\hline 520 & & $\begin{array}{l}\text { LA } \\
\text { U1 }\end{array}$ & $\begin{array}{r}\text { Q2 } \\
-\mathrm{L} 4 \\
\end{array}$ & - F1 & - Q2 \\
\hline 515 & - $F_{1}$ & Q2 & UI & $\begin{array}{l}01 \\
\text { Q }\end{array}$ & F1 \\
\hline $\begin{array}{l}510 \\
505\end{array}$ & $\begin{array}{l}\mathrm{Q}_{2}^{\mathrm{A} 2} \\
\mathrm{U1} \mathrm{L}^{\mathrm{L} 4}\end{array}$ & $\begin{array}{r}1.3 \\
\mathbf{A 2} \\
\mathbf{R} \mathbf{1} \\
\mathbf{Q} \mathbf{1}\end{array}$ & $\begin{array}{l}\text { L2 } \\
\text { L1 } \\
\text { R1 } \\
\text { RI }\end{array}$ & $\begin{array}{l}\mathrm{I} A \\
\mathrm{Q} 2 \\
\mathrm{R} 1 \\
\end{array}$ & $\begin{array}{l}\mathrm{L} 4 \\
\mathrm{~A} 2 \\
\mathrm{R} 1\end{array}$ \\
\hline 500 & $\begin{array}{l}Q_{1} L^{2} 3 \\
V_{1}\end{array}$ & Q VI & Q1 & $\begin{array}{l}\mathrm{L3} \\
\mathrm{V} 1\end{array}$ & $\begin{array}{l}\mathrm{L}_{3} \\
\mathrm{~V} \mathrm{I}\end{array}$ \\
\hline 495 & - 12 & 1.2 & $\bullet \mathbf{L} 2$ & $\cdot \mathrm{L}_{2}$ & $\bullet \mathrm{L}_{2}$ \\
\hline $\begin{array}{l}490 \\
485\end{array}$ & L1 & $\begin{array}{l}\text { L1 } \\
\text { Al } \\
\text { DI }\end{array}$ & $\begin{array}{l}\text { LI } \\
\text { AI } \\
\text { DI }\end{array}$ & $\begin{array}{l}\text { Al } \\
\text { D1 } \\
\text { D1 }\end{array}$ & $\begin{array}{l}\text { Al } \\
\text { : LI } \\
\text { DI }\end{array}$ \\
\hline 480 & D1 & & & & \\
\hline $\begin{array}{l}\text { INI } \\
\text { DE! }\end{array}$ & $\begin{array}{l}\text { TIAL } \\
\text { MAND }\end{array}$ & $\begin{array}{c}\text { RBS } \\
\text { RESULTS }\end{array}$ & $\begin{array}{c}\text { RBSall } \\
\text { RESULTS }\end{array}$ & $\begin{array}{l}\text { MILP GDO } \\
\text { RESULTS }\end{array}$ & $\begin{array}{l}\text { MILPGEA } \\
\text { RESULTS }\end{array}$ \\
\hline $\begin{array}{r}\text { EXEC } \\
\text { C }\end{array}$ & $\begin{array}{l}\text { UTION } \\
\text { OST }\end{array}$ & $\mathrm{C}=201$ & $C=195$ & $C=147$ & $C=134$ \\
\hline
\end{tabular}

Fig. 6. Arrival sequences of flights at EWR: initial demand (blue); and SES results for RBS (yellow), RBSall (green), MILP GDO (red) and MILP G\&A (purple). Data labels indicate simulated flight call signs (airline indicated by a letter, flight by a number). 
TABLE III. COMPARISON OF SCHEDUling MeThods IN TERMS OF EQUiTY

\begin{tabular}{|c|r|c|c|c|c|c|c|c|c|c|c|}
\hline \multirow{2}{*}{ Method: } & Airline: & A & C & D & F & L & Q & R & S & U & V \\
\cline { 2 - 10 } & \% of flights operated & 10.0 & 5.0 & 5.0 & 5.0 & 40.0 & 10.0 & 5.0 & 5.0 & 10.0 & 5.0 \\
\hline \multirow{2}{*}{ RBS } & \% from the total cost & 1.5 & 9.0 & 3.0 & 7.0 & 44.8 & 6.5 & 3.0 & 8.0 & 15.4 & 2.0 \\
\cline { 2 - 11 } & Average cost, min. & 1.5 & $\mathbf{1 8 . 0}$ & 6.0 & $\mathbf{1 4 . 0}$ & 11.3 & 6.5 & 6.0 & $\mathbf{1 6 . 0}$ & $\mathbf{1 5 . 5}$ & 4.0 \\
\hline \multirow{2}{*}{ RBSall } & \% from the total cost & 4.6 & 9.2 & 0.0 & 10.3 & 36.4 & 7.7 & 0.5 & 8.2 & 18.5 & 4.6 \\
\cline { 2 - 11 } & Average cost, min. & 4.5 & $\mathbf{1 8 . 0}$ & 0.0 & $\mathbf{2 0}$ & 8.9 & 7.5 & 1.0 & $\mathbf{1 6 . 0}$ & $\mathbf{1 8 . 0}$ & 9.0 \\
\hline \multirow{2}{*}{ MILP GDO } & \% from the total cost & 22.4 & 3.4 & 2.0 & 4.8 & 25.9 & 20.4 & 1.4 & 2.0 & 17.7 & 0.0 \\
\cline { 2 - 11 } & Average cost, min. & $\mathbf{1 6 . 5}$ & 5.0 & 3.0 & 7.0 & 4.8 & $\mathbf{1 5 . 0}$ & 2.0 & 3.0 & 13.0 & 0.0 \\
\hline \multirow{2}{*}{ MILP G\&A } & \% from the total cost & 5.2 & 10.4 & 0.0 & 3.0 & 19.4 & 40.3 & 3.0 & 1.5 & 17.2 & 0.0 \\
\cline { 2 - 11 } & Average cost, min. & 3.5 & $\mathbf{1 4 . 0}$ & 0.0 & 4.0 & 3.3 & $\mathbf{2 7 . 0}$ & 4.0 & 2.0 & 11.5 & 0.0 \\
\hline
\end{tabular}

\section{Efficiency and equity trade-off within TOS allocation}

In this section, the equity of the proposed solutions is studied from the airline perspective. Table III presents some equity metrics for the test case described in Section V-A, for the 10 simulated airlines. For each airline, it lists the percentage of flights operated (flight share), followed by the percentage of the total system execution cost incurred by that airline (cost share), and the corresponding average cost (in minutes of delay) for each of the scheduling methods. The complete equity can be considered to be achieved if these average costs are equal for all airlines. The highest average costs for each of the methods are highlighted in bold. For the RBS methods, these costs are incurred by airlines $\mathrm{C}, \mathrm{F}, \mathrm{S}$ and $\mathrm{U}$, which do not generally represent the airlines with the highest flight share. However, their flights have late IATs, putting them at the end of the RBS priority list (see Fig. 6, left scale). Airline L also has flights with late IATs, and while its total cost share is high (about $40 \%$ ), its average cost is low thanks to the flights scheduled at the beginning of the list with zero cost. When the equity metric is not taken into consideration during optimization $(\omega=0)$, there is some degree of randomness in the way that the MILP methods assign costs to airlines: MILP GDO yields the best results in terms of equity with maximum average airline cost equal to 16.5 minutes (even better than RBS), while the equity of MILP G\&A solution is the worst with maximum average airline cost being 27 minutes (see Table III). However, these results can be significantly improved when the equity metric, i.e., maximum average airline cost, $y$, is included into the objective function.

Fig. 7 demonstrates the trade-off between system efficiency and equity metrics for different values of $\omega(\alpha=1)$. It is clearly seen for both MILP problems, that as $\omega$ increases, the total system cost increases as well while the maximum average airline cost decreases, with the lowest values achieved for $\omega=5$. In some cases, the gain in equity may be achieved at no expense in efficiency, e.g. with $\omega=1$ for MILP GDO. The range between

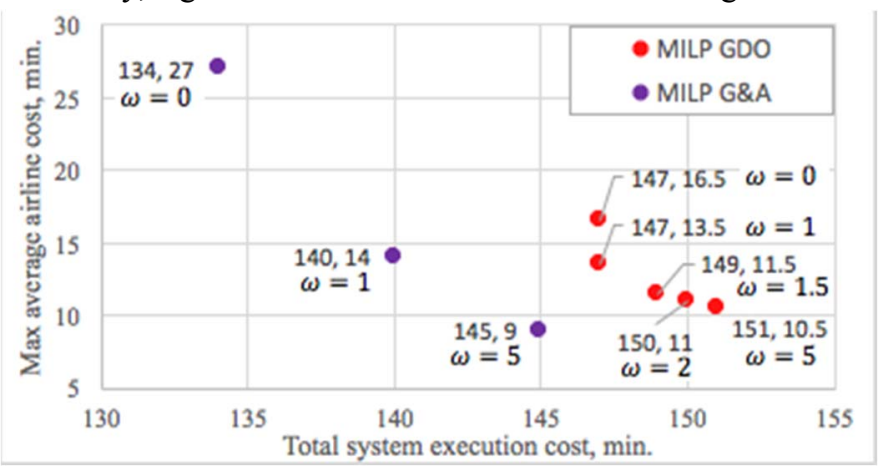

Fig. 7. System efficiency and equity trade-off in MILP results for different values of trade-off coefficient. minimum and maximum values for the efficiency and equity metrics is much wider for MILP G\&A than for MILP GDO; thus, the price of equity is higher for MILP G\&A. However, MILP G\&A still outperforms MILP GDO in terms of efficiency, and also yields the least possible maximum average airline cost (equal to 9 minutes). The best trade-off between efficiency and equity seems to be achieved by MILP G\&A with $\omega=1$. The corresponding metrics values are significantly better than those achieved by the classical RBS method: 140 versus 201 minutes for total system execution cost (see Table II); and 14 versus 18 minutes for maximum average airline cost (see Table III).

\section{SUMMARY}

This paper aims to allocate routes from TOSs and delays to flights within CTOP so as to satisfy the airline preferences and the minimum required spacing between the flights at each FCA, taking into account system efficiency and equity criteria. Two different approaches are studied: an RBS scheme, including classical RBS, which takes into account the constraints at the primary FCA only, and a modification, RBSall, which simultaneously considers constraints at all consecutive FCAs; and global optimization, including a version with ground delays only, MILP GDO, and a version supporting airborne delays as well, MILP G\&A. The methods are compared for a test case involving 4 FCAs and 20 flights destined for EWR, with up to 3 trajectory options each.

The simulation results demonstrate that, in the deterministic case, total system cost can be reduced by taking into account constraints at consecutive FCAs simultaneously, while the schedule becomes more predictable in execution. Global optimization yields solutions with much lower total system cost than the RBS methods. Considering airborne delays in the optimization enables further cost reduction. Including an equity metric in the objective function can improve equity from the airline perspective with a relatively little loss in efficiency. To conclude, MILP G\&A method with a trade-off between efficiency and equity is found to yield the best results for this deterministic TOS allocation problem.

Ongoing work addresses a more realistic test case involving larger CTOP periods of activity and a larger set of flights. The MILP solver computation time becomes an issue in this case, so alternative resolution methods are being considered. Furthermore, predictability of the proposed allocation method is being investigated under varying FCA capacities. Future work will develop a stochastic formulation of the MILP G\&A and compare its performance in terms of efficiency, equity and predictability with corresponding results generated using capacity-based and slot-based allocations. Exempted and pop- 
up flights will also be considered. Ultimately this work will target the development of a reliable scheduling method which can replace the RBS in CTOP.

\section{ACKNOWLEDGMENTS}

This research was supported by an appointment to the NASA Postdoctoral Program at NASA Ames Research Center, administrated by Universities Space Research Association through a contract with NASA. The authors would also like to gratefully acknowledge Guodong Zhu and Prof. Peng Wei for their assistance in improving the optimization computation speed, and helpful discussions.

\section{REFERENCES}

[1] "Traffic Flow Management in the National Airspace System", FAA National Headquarters, Washington, D.C., 2009.

[2] Federal Aviation Administration, NextGen, FAQ: Weather delays, Online: https://www.faa.gov/nextgen/programs/weather/faq/

[3] United States Department of Transportation, Bureau of Transportation Statistics: Understanding the reporting of causes of flight delays and cancellations, Online: https://www.rita.dot.gov/bts/help/aviation/html/understanding.html

[4] National Business Aviation Association, Tools used for Traffic Flow Management, Online: https://www.nbaa.org/ops/airspace/tfm/tools

[5] "CTOP, Collaborative Trajectory Options Program, System Operations Programs", Federal Aviation Administration, Washington, D.C., 2010.

[6] P. Smith, E. Stellings, "Operating in a CTOP (Collaborative Trajectory Options Program) environment", Tech. Rep., 2014.

[7] "TFMS Functional Description, Appendix C: Traffic Management Initiative (TMI) Algorithms", CSC/TFMM-13/1744, Tech. Rep., 2014.

[8] R. Hoffman, J. Burke, T. Lewis, A. Futer, and M. Ball, "Resource allocation principles for airspace flow control", AIAA Guidance, Navigation, and Control Conference and Exhibit, San Francisco, CA, USA, 2005.

[9] C. Wanke, L. Song, S. Zobell, D. Greenbaum, and S. Mulgund, "Probabilistic congestion management", $6^{\text {th }}$ USA/Europe ATM R\&D Seminar, Baltimore, MD, USA, 2005.

[10] R. Hoffman, G. Davidson, and K. Streety, "The equity practices of Collaborative Decision Making in Air Traffic Management", Metron Aviation, Inc., Herndon, VA, USA, Tech. Rep., 2003.

[11] T. Vossen, and M. Ball, "Optimization and mediated bartering models for ground delay program", Naval Research Logistics, Vol. 53, pp. 75-90, 2006.

[12] B. Ye, L. Sherry, M. Hu, and C.H. Chen, "Analysis of alternative collaborative route selection strategies based on cost and throughput", Integrated Communications, Navigation and Surveillance Conference, Herndon, VA, USA, 2012.

[13] M. Ball, R. Hoffman, and A. Mukherjee, "Ground delay program planning under uncertainty based on the ration-by-distance principle", Journal of Transportation Science, vol. 44, No. 1, pp. 1-14, 2010.

[14] National Severe Weather Playbook, FAA, Online: https://www.fly.faa.gov/PLAYBOOK/pbindex.html

[15] Air Traffic Control System Command Center, CDM Operational Coded Departure Routes Database Query, FAA, Online: https://www.fly.faa.gov/rmt/cdm_operational_coded_departur.jsp

[16] A. Bombelli, A. Segarra Torne, E. Trumbauer, and K.D. Mease, "Automated route clustering for air traffic modeling", AIAA Modeling and Simulation Technologies Conference, Grapevine, TX, USA, 2017.

[17] R. Jakobovits, J. Krozel, and S. Penny, "Ground Delay Programs to address weather within en eoute Flow Constrained Areas", AIAA Guidance, Navigation, and Control Conference and Exhibit, San Francisco, CA, USA, 2005.

[18] S. Grabbe, B. Sridhar, and A. Mukherjee, "Sequential traffic flow optimization with tactical flight control heuristics", Journal of Guidance, Control, and Dymanics, vol. 32, No. 3, pp. 810-820, 2009.
[19] M. Ganji, D. Lovell, and M. Ball, "Resource allocation in flowconstrained areas with stochastic termination times considering both optimistic and pessimistic reroutes", $8^{\text {th }}$ USA/Europe ATM R\&D Seminar, Napa, CA, USA, 2009.

[20] A. Mukherjee, S. Grabbe, and B. Sridhar, "Flight departure delay and rerouting under uncertainty in en route convective weather", AIAA Guidance, Navigation, and Control Conference and Exhibit, Portland, OR, USA, 2011.

[21] H.D. Sherali, R.W. Staats, and A.A. Trani, "An airspace planning and collaborative decision-making model: Part II - Cost model, data consideration, and computations", Transportation Science, vol. 40, No. 2, pp. 147-164, 2006.

[22] D. Bertsimas, V.F. Farias, and N. Trichakis, "On the efficiency-fairness trade-off", Management Science, vol. 58, No. 12, pp. 2234-2250, 2012.

[23] J. Henderson, H. Idris, R. Kicinger, J. Krozel, D. Wang, and K. Sheth, "Airline and service provider collaborative algorithms for flight route and delay decisions", AIAA Guidance, Navigation, and Control Conference and Exhibit, Chicago, IL, USA, 2009.

[24] M. Ball, R. Hoffman, D.J. Lovell, and A. Mukherjee, "Response mechanisms for dynamic air traffic flow management", $6^{\text {th }}$ USA/Europe ATM R\&D Seminar, Baltimore, MD, USA, 2005.

[25] A. Mukherjee, S. Grabbe, and B. Sridhar, "Arrival flight scheduling through departure delays and reroutes", Air Traffic Control Quarterly, vol. 17, No. 3, pp. 223-244, 2009.

[26] D.S. Fearing, "Network fairness in Air Traffic Flow Management", Massachusetts Institute of Technology, Cambridge, MA, USA, Tech. Rep., 2007.

[27] J. Cox, and M.J. Kochenderfer, "Optimization approaches to the single airport Ground-Holding Problem", Journal of Guidance, Control, and Dynamics, vol. 38, No. 12, pp. 2399-2406, 2015.

[28] D. Bertsimas, and S. Stock Patterson, "The Traffic Flow Management rerouting problem in Air Traffic Control: A dynamic network flow approach", Transportation Science, vol. 34, No. 3, pp. 239-255, 2000.

[29] M. Bloem, and B. Sridhar, "Optimally and equitably distributing delays within the aggregate flow model", $27^{\text {th }}$ Digital Avionics Systems Conference, Saint Paul, MN, USA, 2008.

[30] H. Arneson, and M. Bloem, "A method for scheduling air traffic with uncertain en route capacity constraints", AIAA Guidance, Navigation, and Control Conference and Exhibit, Chicago, IL, USA, 2009.

[31] J. Le Ny, and H. Balakrishnan, "Feedback control in the National Airspace System", Journal of Guidance, Control, and Dynamics, vol. 34, No. 3, pp.832-846, 2011.

[32] W. Hall, "Efficient capacity allocation in a collaborative air transportation system", Massachusetts Institute of Technology, PhD Thesis, 1999.

[33] J. Burke, "Implementing and evaluating alternative airspace rationing method", National Center of Excellence in Aviation Operations Research (NEXTOR), Master's Thesis, 2002.

[34] A. Kim, and M. Hansen, "A framework for the assessment of collaborative enroute resource allocation strategies", Transportation Research, Part C: Emerging Technologies, vol. 33, pp. 324-339, 2013.

[35] B. Kim, and J.P. Clarke, "Optimal airline actions during Collaborative Trajectory Options Program", AGIFORS 54 ${ }^{\text {th }}$ Annual Symposium, Dubai, UAE, 2014.

[36] D. Bertsimas, and S. Gupta, "A proposal for network Air Traffic Flow Management incorporating fairness and airline collaboration", submitted to Operations Research, 2011.

[37] L. Cruciol, J.P. Clarke, and L. Weigang, "Trajectory option set planning optimization under uncertainty in CTOP", IEEE 18th International Conference on Intelligent Transportation Systems, Las Palmas de Gran Canaria, Canary Islands, Spain, 2015.

[38] A. Evans, V. Vaze, and C. Barnhart, "Airline-driven performance-based Air Traffic Management: Game theoretic models and multicriteria evaluation", Transportation Science, vol. 50, No. 1, pp. 180-203, 2016.

[39] D. Bertsimas, V.F. Farias, and N. Trichakis, "The Price of Fairness", Operations Research, vol. 59, No. 1, pp. 17-31, 2011.

[40] C. Barnhart, D. Bertsimas, C. Caramanis, and D. Fearing, "Equitable and Efficient Coordination in Traffic Flow Management", Journal of Transportation Science, vol. 46, No. 2, pp. 262-280, 2012. 